extreme heterogeneity and often includes patients with coexisting pathologies, one obvious example being asthma and emphysema. The assumption that it defines anything resembling a cohesive entity in terms of aetiology or pathogenesis is anti-intellectual, and the obvious bias in studies of this nature is just one example. The demonstration that inhaled corticosteroids are effective in "COPD" defined in this way tells us nothing about their efficacy (or more likely lack of it) in smoking-related airflow limitation. "COPD" should be the acronym for "Cop-out On Proper Diagnosis". Not only does it confound the proper assessment of common disease processes, it prevents the effective evaluation of rarer ones, and probably the recognition of new and important entities; no wonder $\alpha$-1 antitrypsin-deficient emphysema was discovered by a biochemist, not a clinician.

\section{P. Barber}

Bronchology Unit, Wythenshawe hospital, Manchester, UK.

\section{References}

1. Suissa S. Inhaled steroids and mortality in COPD: bias from unaccounted immortal time. Eur Respir J 2004; 23: 391-395.

DOI: $10.1183 / 09031936.04 .10062504$

\section{From the authors:}

P. Barber correctly points out the difficulties in identifying chronic obstructive pulmonary disease (COPD) patients using administrative databases, and especially in distinguishing between COPD and asthma. This is particularly important when assessing the impact of inhaled corticosteroids because these drugs have been shown in randomised trials to be extremely effective in asthma but not in COPD. Thus, a study based on a mixed population that includes both asthma and COPD patients will result in an average effect for inhaled corticosteroids.

To maximally ensure the accuracy of a first-time COPD diagnosis for cohort entry, we used three criteria: 1) 55 yrs of age or over; 2) three or more prescriptions on at least two different dates for a bronchodilator within a 1-yr period; and 3) no other prescriptions for bronchodilators or other asthma drugs during the 5-yr period prior to the three defining prescriptions $[1,2]$. With these criteria, we were confident that the proportion of asthma patients would be kept to a negligible level.

In our previous research on asthma, we effectively used similar criteria to study asthma, with the difference that age at cohort entry (diagnosis) was restricted to between 5 and 44 yrs, thus reducing the possibility of including COPD patients [3-5].

While observational databases studies are challenging, they are indispensable to complement other types of studies into the effects of drugs. As P. Barber notes, scientific rigor must be introduced in their design and analysis, which can be best achieved by intense collaborations between clinicians and methodologists. We have and will continue to work in this direction.

\section{S. Suissa}

Division of Clinical Epidemiology, McGill University, Royal Victoria Hospital, Montreal, Quebec, Canada.

\section{References}

1. Suissa S, Assimes T, Ernst P. Inhaled short acting beta agonist use in COPD and the risk of acute myocardial infarction. Thorax 2003; 58: 43-46.

2. de Melo MN, Ernst P, Suissa S. Inhaled corticosteroids and the risk of a first exacerbation in COPD patients. Eur Respir $J$ 2004; 23: 692-697.

3. Suissa S, Ernst P, Benayoun B, Baltzan M, Cai B. Low-dose inhaled corticosteroids and the prevention of death from asthma. N Engl J Med 2000; 343: 332-336.

4. Suissa S, Ernst P, Kezouh A. Regular use of inhaled corticosteroids and the long term prevention of hospitalisation for asthma. Thorax 2002; 57: 880-884.

5. Suissa S, Assimes T, Brassard P, Ernst P. Inhaled corticosteroid use in asthma and the prevention of myocardial infarction. Am J Med 2003; 115: 377-381.

\title{
Protective effect on AMP airway responsiveness after a single dose of fluticasone
}

\section{To the Editor:}

We read with great interest the article by LuIJK et al. [1] describing the time-course of substantial protective effects after a single dose of inhaled fluticasone propionate (FP) on adenosine-5'-monophosphate (AMP)-induced bronchoconstriction in asthma. No change was observed in terms of exhaled nitric oxide (eNO) levels.

These findings would further support the current opinion that airway responsiveness to AMP is more sensitive than eNO, together with other noninvasive markers of airway inflammation, in assessing the response to anti-inflammatory treatments [2, 3]. To this end, it must be emphasised that inhaled glucocorticosteroids (GCS) have been shown to increase the provocative concentration causing a $20 \%$ fall in forced expiratory volume in one second (PC20) threshold of AMP in a dose-dependent manner, whereas the dose response for eNO, together with many other inflammatory markers, and measures of lung function have all been shown to exhibit a plateau effect at lower doses [4]. Thus, the changes observed with eNO are not truly dose dependent, whereas the degree of anti-inflammatory effects of AMP challenge are directly proportional to the dose of inhaled GCS. The idea that AMP bronchial provocation seems to offer substantial advantages over eNO has been recently supported by the work of PRIETO et al. [5], in which airway responsiveness to inhaled AMP is a useful predictor for safe dose reduction of inhaled GCS in patients with asthma. 
Whereas the previously mentioned studies appear to provide enough information to support an important role for AMP provocation in the clinical setting, the study by LUIJK et al. [1] raises some important concerns.

Although the rapid protective effect by a single dose of inhaled GCS has never been studied in asthmatic subjects receiving chronic treatment with GCS, the extreme sensitivity of AMP to these drugs could be detrimental to its potential clinical applications, where it is crucial to assess the dose requirement of topical GCS in asthma management. This is not a trivial issue, since the acute effect on AMP challenge is not specific to FP, but it also appears to be shared with other common inhaled GCS, including beclomethasone and budesonide [6].

Therefore, if we want to investigate adenosine-5'-monophosphate for monitoring inhaled glucocorticosteroid requirements in clinical asthma and establishing the appropriate dose needed to control airway inflammation, patients should refrain from taking steroids for $\geqslant 24-48 \mathrm{~h}$ prior to adenosine monophosphate AMP challenge (as its readout: the provocative concentration of adenosine monophosphate causing a $20 \%$ fall in forced expiratory volume in one second could be significantly influenced by the last inhaled dose of glucocorticosteroids).

\section{Spicuzza, G. Di Maria, R. Polosa}

Dept of Internal Medicine and Medical Specialities, University of Catania, Catania, Italy.

\section{References}

1. Luijk B, Kempsford RD, Wright AM, Zanen P, Lammers JJ Duration of effect of single-dose inhaled fluticasone propionate on AMP-induced bronchoconstriction. Eur Respir $J$ 2004; 23: 559-564.

2. Prosperini $\mathrm{G}$, Rajakulasingam $\mathrm{K}$, Cacciola $\mathrm{RR}$, et al. Changes in sputum counts and airway hyperresponsiveness after budesonide: monitoring anti-inflammatory response on the basis of surrogate markers of airway inflammation. J Allergy Clin Immunol 2002; 110: 855-861.

3. Spicuzza L, Bonfiglio C, Polosa R. Research applications and implications of adenosine in diseased airways. Trends Pharmacol Sci 2003; 24: 409-413.

4. Wilson AM, Lipworth BJ. Dose-response evaluation of the therapeutic index for inhaled budesonide in patients with mild-to-moderate asthma. Am J Med 2000; 108: 269275.

5. Prieto L, Bruno L, Gutierrez V, et al. Airway responsiveness to AMP and exhaled nitric oxide measurements. Predictive value as markers for reducing the dose of inhaled corticosteroids in asthmatic subjects. Chest 2003; 124: 1325-1333.

6. Ketchell RI, Jensen MW, Clark GW, Allenby MI, O'Connor BJ. Rapid effect of single dose inhaled glucocorticosteroid on airway hyperresponsiveness to AMP in asthma is not molecule dependent. Am J Respir Crit Care Med 2003; A770: D54 (abstract). 